\title{
Photon side-bands in mesoscopics *
}

\author{
A. P. Jauho \\ Mikroelektronik Centret, Technical University of Denmark, Bldg. 345 e \\ DK-2800 Lyngby, Denmark
}

(May 23, 2021)

\begin{abstract}
This paper reviews several applications of photonic side-bands, used by Büttiker and Landauer in their theory of traversal time in tunneling [Phys. Rev. Lett. 49, 1739 (1982)], in transport and optics of mesoscopic systems. Topics include generalizations of the transmission theory of transport to time-dependent situations, optics and transport of mesoscopic systems in $\mathrm{THz}$ electromagnetic fields, and phase-measurements of photon-assisted tunneling through a quantum dot.
\end{abstract}

\section{INTRODUCTION}

In 1982 Markus Büttiker and Rolf Landauer published a paper on traversal times in tunneling [ [1], that rekindled the interest in an old topic and served as an inspiration for much subsequent research. Several articles in this volume address the recent developments in this field, which embraces a wide scope ranging from foundations of quantum theory to practical questions concerning ultimate speed limits of nanoelectronic components, a very characteristic feature of Rolf Landauer's research. The point of the present article, however, is not to participate in that particular discussion, but rather to use a technical device presented in the 1982-paper to analyze three other physical systems all of which have connections to problems Rolf Landauer has been active in.

The idea of Büttiker and Landauer was to study the sensitivity of tunneling transmission coefficient through a potential barrier to a time-periodic perturbation imposed on the barrier. As the frequency of the modulation is varied, the inverse of a cross-over frequency was identified as a characteristic time-scale for tunneling. The solution to the time-dependent Schrödinger equation in the barrier region was written, following Tien and Gordon [2], as

$$
\psi_{ \pm}(x, t, E)=e^{ \pm \kappa x} e^{-i E t / \hbar} \exp \left(-\frac{i V_{1}}{\hbar \omega} \sin \omega t\right)
$$

*An article to commemorate Rolf Landauer's 70th birthday. 


$$
=e^{ \pm \kappa x} e^{-i E t / \hbar}\left[\sum_{n=-\infty}^{n=+\infty} J_{n}\left(\frac{V_{1}}{\hbar \omega}\right) e^{-i n \omega t}\right],
$$

where $V_{1}$ is the amplitude of the harmonic time-modulation, $\omega$ is its frequency and $\kappa=$ $\left\{2 m\left[V_{0}-E\right]\right\}^{1 / 2} / \hbar$ with $V_{0}$ the barrier height. The amplitudes of the side-bands at energies $E \pm n \hbar \omega$ are given by the Bessel functions $J_{n}$. Since the time-modulation was introduced just as formal device to probe the energy sensitivity of transmission, Büttiker and Landauer were interested in the weak perturbation limit, $V_{1} / \hbar \omega \ll 1$. In this paper, on the other hand, we ask the question: What are the consequences of Eq.(11) in physical situations where the external perturbation is not weak? Several examples will be discussed below. In real physical systems interactions (impurities, phonons, electron-electron collisions) must be considered, and the interaction terms must be included in the Hamiltonian. It is often convenient to formulate the analysis in the language of many-body formalism, and instead of working directly with Eq.(11), we prefer to use the spectral function, which now reads

$$
A\left(\mathbf{p}, t, t^{\prime}\right)=\exp \left[-i \int_{t^{\prime}}^{t} d t_{1} \epsilon\left(\mathbf{p}, t_{1}\right)\right]
$$

Note that the time-dependent perturbation requires one to move away from the conventional energy representation, $A(\mathbf{p}, E)=2 \pi \delta(E-\epsilon(\mathbf{p}))$. Further, the above expression is a slight generalization of Eq.(1) in that by an appropriate choice of the time-dependent singleparticle energy $\epsilon(\mathbf{p}, t)$ several different physical systems can be addressed, e.g., with $\epsilon(\mathbf{p}, t)=$ $\epsilon(\mathbf{p}-q \mathbf{A}(t))$, where $\mathbf{A}(t)$ is the vector potential, a uniform time-dependent electric field may be included, while setting $\epsilon(\mathbf{p}, t)=p^{2} / 2 m+V_{1} \cos \omega t$ we return to situation discussed in Ref.[1], as is readily verified by doing the integral in Eq.(2), and expanding the result in terms of Bessel functions, in full analog with Eq.(11). We now proceed to the applications.

\section{TIME-DEPENDENT TRANSPORT IN MESOSCOPIC SYSTEMS}

In order that the consequences of Eqs.(14,21) be visible in an experiment at least the following two conditions must be met. First, the system must maintain a certain degree of phase-coherence during a significant part of the transport process. Second, the timevariation must be different in different parts of the system, and the particles must be able to move between these regions. This brings us in the realm of mesoscopic systems. Now, the transport in mesoscopic systems can be analyzed extremely successfully in terms of conductance formulas, pioneered by Landauer [ [3], and their subsequent generalizations [ 4 ]. Generically, one can express the conductance of a mesoscopic system, coupled by ideal leads to external reservoirs, as

$$
g=\frac{e^{2}}{h} T\left(\epsilon_{F}\right)
$$

where $T=|t|^{2}$ is the transmission coefficient, and $t$ is the complex transmission amplitude. But here we are addressing a problem going beyond the original formulation: we have an external time-dependence acting on the system. Further, it would be desirable to include 
interactions within the mesoscopic regions: these can be very important due to the small number of charge carriers which implies less effective screening.

Recent years have witnessed a flurry of theoretical papers reporting on generalizations of the original scattering-approach to interacting and/or time-dependent systems. It would be beyond the present purposes to provide a complete list of references, instead we point to two chapters [ 5, 6], representing complementary views, in a forthcoming volume in Handbook of Semiconductors. The formulation we adopt here focuses on the tunneling part of the current! and the current from the left reservoir to the mesoscopic region (we focus on a two-terminal geometry) can be expressed as [5]

$$
J_{L}(t)=-\frac{2 e}{\hbar} \int_{-\infty}^{t} d t_{1} \int \frac{d \epsilon}{2 \pi} \operatorname{Im} \operatorname{Tr}\left\{e^{-i \epsilon\left(t-t_{1}\right)} \boldsymbol{\Gamma}^{L}\left(\epsilon, t, t^{\prime}\right)\left[\mathbf{G}^{<}\left(t, t_{1}\right)+f_{L}(\epsilon) \mathbf{G}^{r}\left(t, t_{1}\right)\right]\right\}
$$

Here the bold-face entities are matrices in the quantum numbers specifying the states in the mesoscopic region; $\Gamma^{L}$ is the coupling matrix to the left reservoir (the time-dependence may be due to external gates which modify the potential barriers between leads and the mesoscopic region) and the Green functions $\mathbf{G}^{<, r}$ must be calculated in the presence of the coupling to the leads. Typically, one would use the Dyson equation to calculate $\mathbf{G}^{r}$ while $\mathbf{G}^{<}$ requires the use of a quantum kinetic equation, e.g. the Keldysh equation. Thus, Eq.(任) is a formal expression for the time-dependent current, nevertheless it appears to form a suitable starting point for further calculations, such as those reported in Refs. [ 7, 8, 9].

Many experiments focus on the average current and it is therefore natural to ask whether Eq.(荬 could be simplified in this case. Indeed, one finds

$$
\left\langle J_{L}(t)\right\rangle=-\frac{2 e}{\hbar} \int \frac{d \epsilon}{2 \pi}\left[f_{L}(\epsilon)-f_{R}(\epsilon)\right] \operatorname{Im} \operatorname{Tr}\left\{\frac{\Gamma^{L}(\epsilon) \boldsymbol{\Gamma}^{R}(\epsilon)}{\Gamma^{L}(\epsilon)+\Gamma^{R}(\epsilon)}\langle\mathbf{A}(\epsilon, t)\rangle\right\},
$$

where $f_{L / R}(\epsilon)=1 /\left[1+\exp \left(\left(\epsilon-\mu_{L / R}\right) / k T\right)\right]$ are distribution functions describing the noninteracting contacts with electro-chemical potential $\mu_{R / L}$, and $\mathbf{A}$ is an object closely related to the retarded Green function [ 10]. This expression is of the Landauer type: it expresses the current as an integral over a weighted density of states times the difference of the two contact occupation factors. There is an important distinction, however: the quantity in curly brackets does not involve, in general, just the transmission coefficient but rather the Green function for the fully interacting system, which must be evaluated in the presence of interactions (e.g. electron-electron, electron-phonon, and spin-flip). The derivation of Eq.(5) allows arbitrary interactions in the mesoscopic region, however the energy dependence of the coupling matrices to right and left contacts must be proportional to each other?.

\footnotetext{
${ }^{1}$ The current measured in the contacts also contains contributions from displacement currents. A low-frequency theory for these, as well as long-range Coulomb forces is described in Ref.[6]

${ }^{2}$ This condition is not too restrictive: in most cases the coupling matrices are assumed to be constants (in lack of a detailed model) and the conditions for the validity of Eq.(5) are automatically satisfied.
} 
Let us now apply these results to a simple example. We consider a single, noninteracting state with energy $\epsilon_{0}$ in the mesoscopic region under the influence of a harmonically varying field with amplitude $V_{1}$. An explicit solution can readily be written down:

$$
\langle A(\epsilon, t)\rangle=\sum_{k=-\infty}^{\infty} J_{k}^{2}\left(\frac{V_{1}}{\hbar \omega}\right) \frac{\Gamma / 2}{\epsilon-\epsilon_{0}-k \omega+i \Gamma / 2} .
$$

Combining Eqs.(5) and the imaginary part of ([6) we find that the current can be written as

$$
\langle J(t)\rangle=\frac{e}{h} \sum_{k=-\infty}^{\infty} \int d \epsilon\left[f_{L}(\epsilon)-f_{R}(\epsilon)\right] T(\epsilon-k \hbar \omega) J_{k}^{2}\left(\frac{V_{1}}{\hbar \omega}\right),
$$

where $T(\epsilon)$ is the elastic transmission coefficient through the mesocopic system. The resulting low-field low-temperature conductance is then

$$
g_{\mathrm{ac}}=\frac{e^{2}}{h} \sum_{k=-\infty}^{\infty} T\left(\epsilon_{F}-k \hbar \omega\right) J_{k}^{2}\left(\frac{V_{1}}{\hbar \omega}\right),
$$

which indeed appears as a natural generalization of the standard conductance Eq.(3) to the time-dependent situation. These expressions bear a very close mathematical resemblance to the results obtained by Tien and Gordon [2] and Tucker [11], who found

$$
\left\langle J\left(V_{0}, t\right)\right\rangle=\sum_{k=-\infty}^{\infty} J_{k}^{2}\left(\frac{V_{1}}{\hbar \omega}\right) I_{\mathrm{dc}}\left(V_{0}-k \hbar \omega / e\right),
$$

i.e., the rectified current of a system biased with $V(t)=V_{0}+V_{1} \cos \omega t$ is given as a sum of dc-currents $I_{\mathrm{dc}}$ evaluated at voltages shifted by integer multiples of photon energies. It is important to note, however, that Eq.(7) was obtained as an explicit calculation for a simple time-dependent resonant level keeping the coupling to equilibrium contacts to all orders, while Refs.[ [ Q, 11] only consider the lowest order coupling between the different parts of the system, but do not make other restrictive assumptions. It would be interesting to learn more about the precise interrelation of these approaches, in particular because Eq.(9) has been very succesful in the analysis of recent experiments on semiconductor superlattices which are subjected to strong ac-fields originating from free electron lasers [ [12, 13, 14].

\section{LINEAR OPTICAL ABSORPTION IN THZ-FIELDS}

Our second application concerns the situation where the system under investigation is placed in a strong THz-field (FIR-field) and then its optical absorption coefficient is measured with a weak probe field in the near infrared part of the spectrum, corresponding to near band-edge absorption in GaAs-based semiconductor systems. As we shall see, the photonic side-band structure residing in Eq.(2) leads to interesting, and observable effects. The dominant features in the measured optical absorption spectrum derive from two basic effects: those due to a modified density of states, and those due to excitons, v.i.z. interaction effects. We analyze both of these effects; what makes our discussion somewhat nonstandard is that we allow arbitrary strength for the THz-field and thus go beyond $\chi^{(3)}$-effects. 


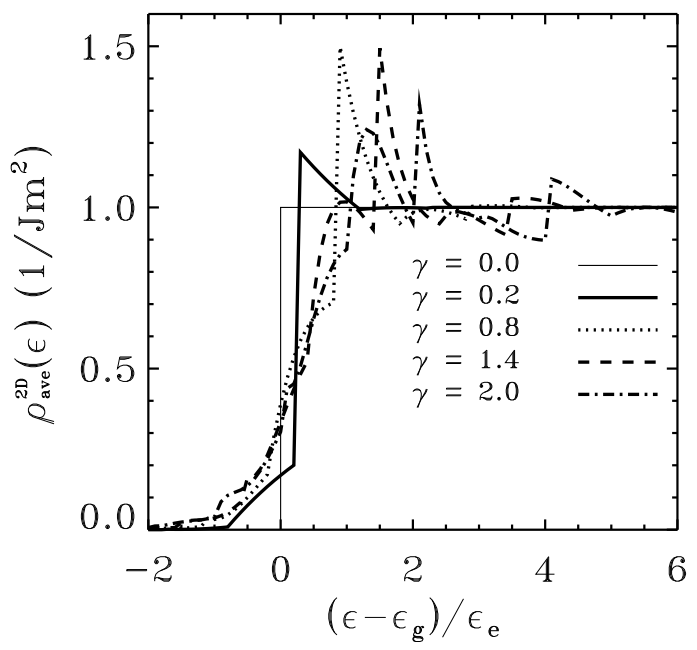

FIG. 1. The time averaged GDOS for a 2D-system for a range of $\mathrm{THz}$ field intensities, parametrized by $\gamma \equiv e^{2} E_{\mathrm{THz}}^{2} / 4 \hbar m^{*} \omega^{3}=(0.2,0.5,0.8,1.1,1.4,1.7,2.0)$, as a function of scaled energy $\left(\epsilon_{e} \equiv \hbar \omega\right)$. At low intensities one observes a Stark-like blue-shift of the band edge as well as finite absorption within the band gap. The blue-shift is given by $\epsilon_{f} \equiv \gamma \omega$, and is physically interpreted as the average kinetic energy of a classical charged particle with mass $m^{*}$ placed in an oscillating electric field of frequency $\omega$ and strength $E_{\mathrm{THz}}$. With increasing intensity side bands emerge at $\epsilon=\epsilon_{g}+\epsilon_{f} \pm 2 \hbar \omega$. (From Ref.[15])

Let us first consider the density of states effects. In a recent paper we have shown [15] that in the noninteracting case the time-dependent absorption coefficient $\alpha_{T}\left(\omega_{l}\right)$, where $\omega_{l}$ is the frequency of the probe field, can be calculated from

$$
\alpha_{T}\left(\omega_{l}\right) \simeq \frac{2 \pi^{2} \omega_{l}|d|^{2}}{c n \hbar} \rho\left(T, \omega_{l}\right)
$$

where $d$ is the dipole matrix element, $n$ is the refraction coefficient, and the generalized density of states [16] is defined in terms of Eq.(2),

$$
\rho\left(T, \omega_{l}\right)=\frac{1}{\pi} \sum_{\mathbf{k}} A\left(\mathbf{k}, T, \omega_{l}\right)
$$

where the Fourier-transform of Eq.(2) is taken with respect to $t-t^{\prime}$, and $T=\left(t+t^{\prime}\right) / 2$. Already this simple result contains some interesting physics. In the dc-limit one recovers the standard Franz-Keldysh absorption spectrum, i.e., a finite (but exponentially damped) absorption in the gap, and characteristic oscillations in the band region. In the time-dependent case, shown in Fig. 1, a dynamical Franz-Keldysh effect occurs [16, 17]: The overall absorption edge experiences a blue-shift, and additional structure appears, both in the gap region and in the continuum.

Excitonic effects require a generalization of the analysis presented above. Rather than just focusing on the density of states, one must evaluate the susceptibility function, $\chi^{r}\left(t, t^{\prime}\right)=$ 


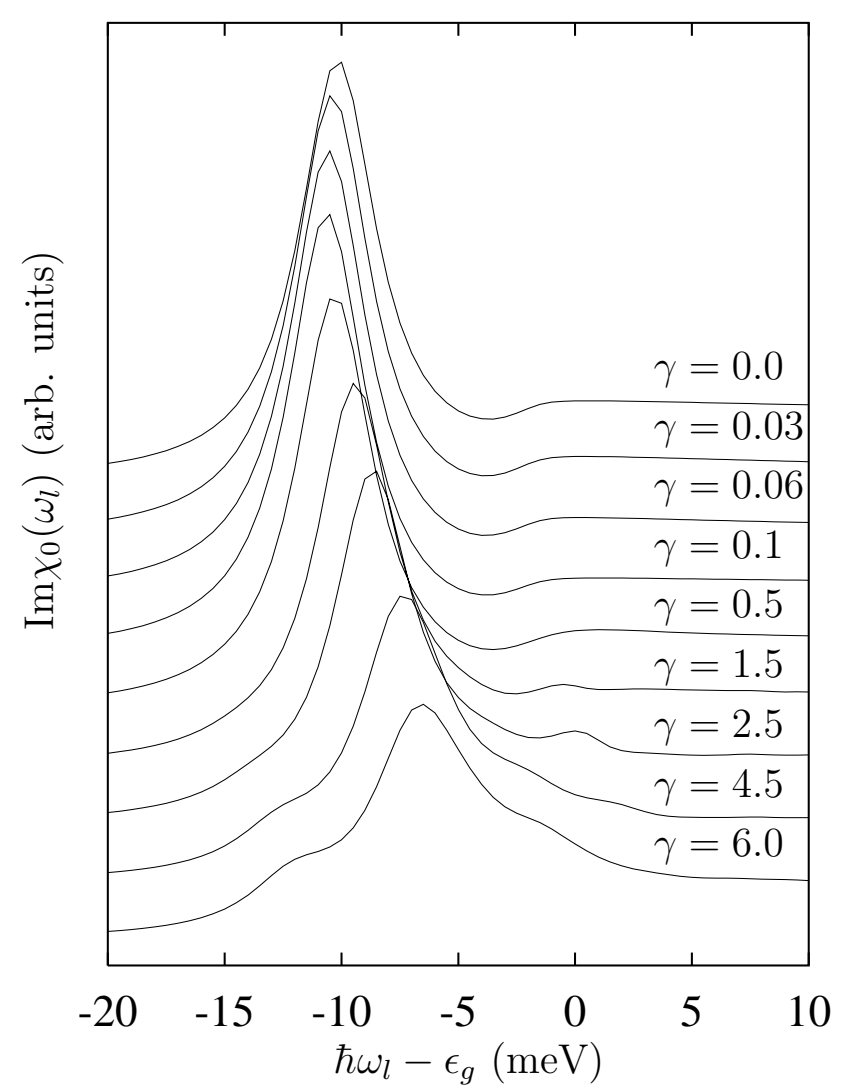

FIG. 2. Linear optical absorption in a quantum well for a range of THz-intensities, parametrized by $\gamma=e^{2} E_{\mathrm{THz}}^{2} / 4 \hbar m^{*} \omega^{3}$. The photon energy is $\hbar \omega=2.5 \mathrm{meV}$, below the ionization threshold. For small $\gamma$ the main feature is red-shifted, while for increasing $\gamma$ a blue-shift, due to the dynamical Franz-Keldysh effect, occurs. Also, exciton replicas at $\pm 2 \hbar \omega$ become visible. (From Ref.[19])

$-i \theta\left(t-t^{\prime}\right)\left\langle\left[P(t), P\left(t^{\prime}\right)\right]\right\rangle$, where $P(t)$ is the polarization. In the noninteracting limit one can show that $\operatorname{Im} \chi_{0}^{r}\left(T, \omega_{l}\right) \propto \rho\left(T, \omega_{l}\right)$, thus establishing a connection to Fig. 1. The Coulomb interaction between the electron and hole can be included by considering the Bethe-Salpeter equation [18], which we have generalized to include the THz-field nonperturbatively. In the photon side-band language the equation reads

$$
\begin{aligned}
\chi_{n}^{r}\left(\mathbf{k}, \omega_{l}+2 n \omega\right) & =\chi_{0, n}^{r}\left(\mathbf{k}, \omega_{l}+2 n \omega\right) \\
& +\sum_{n^{\prime}} \chi_{0, n-n^{\prime}}^{r}\left(\mathbf{k}, \omega_{l}+2\left(n+n^{\prime}\right) \omega\right) \int \frac{d \mathbf{k}}{(2 \pi)^{2}} V\left(\left|\mathbf{k}-\mathbf{k}^{\prime}\right|\right) \chi_{n^{\prime}}^{r}\left(\mathbf{k}, \omega_{l}+2 n^{\prime} \omega\right),
\end{aligned}
$$

where the Fourier representation is defined via

$$
\chi^{r}\left(t, t^{\prime}\right)=\sum_{n} \int \frac{d \omega}{2 \pi} \chi_{n}^{r}(\omega) e^{i \omega_{l}\left(t-t^{\prime}\right)+i n 2 \omega\left(t+t^{\prime}\right)} .
$$

This equation can be solved via standard numerical methods, and the optical absorption coefficient is finally obtained from $\operatorname{Im} \chi_{0} \equiv \sum_{\mathbf{k}} \operatorname{Im} \chi_{n=0}^{r}\left(\mathbf{k}, \omega_{l}\right)$. Typical numerical results are 
shown in Fig. 2. The theoretical predictions can be briefly summarized as follows. If the $\mathrm{THz}$ frequency is smaller than the frequency corresponding to the ionization energy of the exciton (i.e. the energy difference corresponding to $1 s \rightarrow 2 p$ transition), the ac Stark effect leads to the red-shift seen at low intensity curves. However, the dynamical Franz-Keldysh effect of Fig. 1 leads to a blue shift which eventually overcomes the red-shift, and a net blueshift results. Recent measurements performed at the UCSB Free Electron Laser facility, to be fully described elsewhere [20], are in very good agreement with these predictions. On the other hand, if the $\mathrm{THz}$ frequency is larger than the ionization thresold, the ac Stark shift and dynamical Franz-Keldysh effect work in unison, and a blue shift is always observed, both theoretically and experimentally.

\section{PHASE MEASUREMENT OF PHOTON-ASSISTED TUNNELING THROUGH A QUANTUM DOT}

The basic conductance formula, Eq.(3), involves the absolute square of the transmission amplitude. The question is then: Can one measure the phase of the transmission amplitude? An affirmative answer was given by the recent groundbreaking experiments of Yacoby et al. [ 21] and Schuster et al. [ 22]. Their experimental protocol runs as follows: A magneto-transport measurement is performed on an Aharonov-Bohm ring with a quantum dot fabricated in one of its arms. If the quantum dot supports coherent transport, the transmission amplitudes through the two arms interfere. A magnetic field induces a relative phase change, $2 \pi \Phi / \Phi_{0}$, between the two transmission amplitudes, $t_{0}$ and $t_{\mathrm{QD}}$, leading to an oscillatory conductance $g(B)=\left(e^{2} / h\right) \mathcal{T}(B)$, with

$$
\mathcal{T}(B)=\mathcal{T}^{(0)}+2 \operatorname{Re}\left\{t_{0}^{*} t_{\mathbf{Q D}} e^{2 \pi i \Phi / \Phi_{0}}\right\}+\ldots
$$

where $\Phi$ is the flux threading the ring, $\Phi_{0}=h c / e$ is the flux quantum, and where the ellipsis represent higher harmonics due to multiple reflections. In the experiments, an oscillatory magnetoconductance of this form was clearly observed thus demonstrating coherent transmission through the dot [21,22] Furthermore, controlling the potential on the dot with a side-gate voltage, allowed measurement of the phase shift of the transmission amplitude. The success of these experiments gave rise to a number of other works which concentrated on refining the interpretation of the experimental results [23 26]. Yet, the experiments also suggest application to other phase-coherent transport processes. One particular example which has been of considerable recent interest, both experimentally [ 12, 27 30] and theoretically [31 35],8], is photon-assisted tunneling. While photon-assisted tunneling (PAT) is intrinsically a coherent phenomenon, existing measurements of PAT are insensitive to the phase of the transmitted electrons and do not directly demonstrate coherence in the presence of the time-dependent field. We have recently proposed [38] a measurement of photon-assisted tunneling through a quantum dot in the mesoscopic double-slit geometry described above. This is, in essence, a combination of the experiments of Kouwenhoven et al. [28,30] where a microwave modulated side-gate voltage gave rise to photon-assisted tunneling through a quantum dot, and the interference experiments of [21] and [22].

We focus on transport in the neighborhood of a single Coulomb oscillation peak associated with a single nondegenerate electronic level of the quantum dot [36]. The effect of the 
ac side-gate voltage is described entirely through the time-dependent energy of this level

$$
\epsilon(t)=\epsilon_{0}\left(V_{\mathrm{s}}\right)+V_{1} \cos \omega t,
$$

i.e., precisely of the type considered in above. Now we also emphasize that the static energy of the level $\epsilon_{0}$ depends on the dc side-gate voltage $V_{\mathbf{s}}$. All other levels on the dot can be neglected provided the ac amplitude, $V_{1}$, and the photon energy, $\hbar \omega$, are small compared to the level spacing on the dot.

In the absence of an ac potential, a suitable model for the transmission amplitude $t_{\mathrm{QD}}(\epsilon)$ through the dot is the Breit-Wigner form,

$$
t_{\mathrm{QD}}(\epsilon)=\frac{-i \sqrt{\Gamma_{L} \Gamma_{R}}}{\epsilon-\epsilon_{0}\left(V_{\mathrm{s}}\right)+i \Gamma / 2}
$$

where $\Gamma=\Gamma_{L}+\Gamma_{R}$ is the full width at half maximum of the resonance on the dot due to tunneling to the left and right leads. Eq. (16) implies a continuous phase accumulation of $\pi$ in the transmission amplitude as the Coulomb blockade peak is traversed. (Note that the Breit-Wigner form is exact for a noninteracting system with $\Gamma$ independent of energy.)

In the dynamic case, the simple Breit-Wigner description must be generalized, and the object to evaluate is the $S$-Matrix element [ [10,37]. Provided interactions in the leads can be neglected, the elastic transmission amplitude $t_{\mathrm{QD}}(\epsilon)$ can be written as the energy conserving part of the $S$-Matrix between the left lead and the right lead

$$
\lim _{\epsilon^{\prime} \rightarrow \epsilon}\left\langle\epsilon^{\prime}, R|\mathcal{S}| \epsilon, L\right\rangle=\delta\left(\epsilon^{\prime}-\epsilon\right) t_{\mathrm{QD}}(\epsilon) .
$$

The $S$-Matrix is simply related to the retarded Green function of the level on the dot, including both tunneling to the leads and the ac potential [ 37], and we find [ 38]

$$
t_{\mathrm{QD}}(\epsilon)=-i \sqrt{\Gamma_{L} \Gamma_{R}}\langle A(\epsilon, t)\rangle .
$$

In the spirit of the Breit-Wigner transmission amplitude we can use the noninteracting $\langle A(\epsilon, t)\rangle$ given by Eq.(6) in further calculations. At finite temperatures one must compute $t_{\mathrm{QD}}=\int d \epsilon\left(-\partial f_{0} / \partial \epsilon\right) t_{\mathrm{QD}}(\epsilon)$ where $f_{0}(\epsilon)$ is the Fermi function, and the final result is

$$
t_{\mathrm{QD}}=\left(-\frac{\Gamma}{4 \pi T}\right) \sum_{k=-\infty}^{\infty} J_{k}^{2}\left(V_{\mathrm{ac}} / \hbar \omega\right) \psi^{\prime}\left[\frac{1}{2}-\frac{i}{2 \pi T}\left(\mu-\epsilon_{0}\left(V_{\mathrm{s}}\right)-k \hbar \omega+i \frac{\Gamma}{2}\right)\right],
$$

where $\psi^{\prime}$ is the derivative of the digamma function, and $\mu$ is the chemical potential in the leads.

We emphasize that a conventional conductance measurement would yield information only about the time average of the square of the transmission amplitude, and the doubleslit geometry of Ref.[21,22] is necessary in order to probe the phase. Figure 3 shows the computed magnitude of $t_{\mathrm{QD}}$ (bottom) and its phase (top), as a function of the level energy $\epsilon_{0}\left(V_{\mathrm{s}}\right)$. As compared to the time-independent case (shown as a dotted line), several features are noteworthy. The magnitude of $t_{\mathrm{QD}}$ shows photonic side-bands, reminiscent of those seen in transmission through a microwave modulated quantum dot [28]. However, there is 


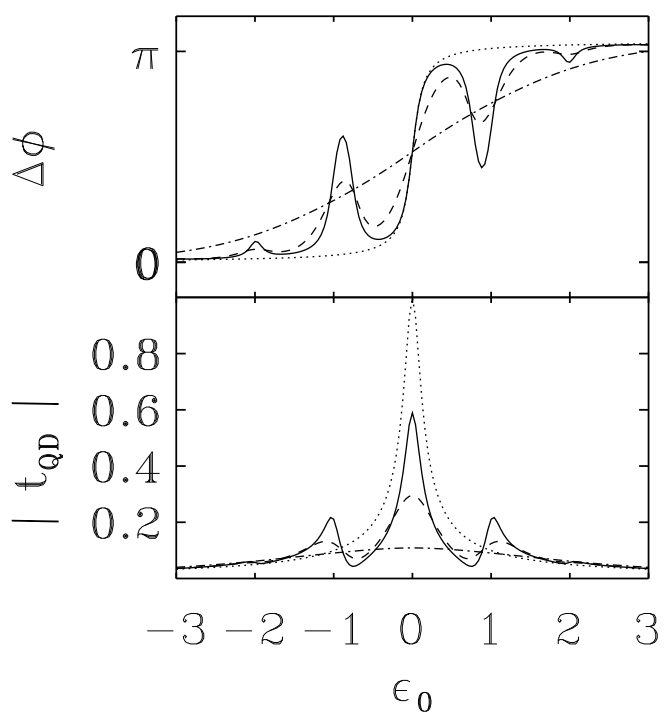

FIG. 3. Temperature dependence of the phase shift $\Delta \phi$ (top panel) and the square of the amplitude (bottom) of $t_{\mathrm{QD}}$. The level-width is $\Gamma / 2=0.1$, in terms of which the other parameters are $V_{1}=1.0, \omega=1.0$, and $T=0$ (solid line), 0.1 (dashed line), 0.5 (dash-dotted line). For comparison, the $T=0$ time-independent results are shown as dots. (From Ref.[39])

an important difference from the usual case of photon-assisted tunneling. The amplitude of the Aharonov-Bohm oscillation is sensitive only to the time average of the transmission amplitude $t_{\mathrm{QD}}$. Hence only elastic transmission through the dot contributes, $i$. $e$., the net number of photons absorbed from the ac field must be zero. The sideband at say $\epsilon=\epsilon_{0}\left(V_{s}\right)-\hbar \omega$ corresponds to a process in which an electron first absorbs a photon to become resonant at energy $\epsilon_{0}\left(V_{s}\right)$, and subsequently reemits the photon to return to its original energy. In Ref.[38] we have studied the phase as a function of the strength of the time-dependent modulation, and find that it is possible to quench the main transmission peak, or change the sign of the slope or the phase at resonance by adjusting the ratio $V_{1} / \hbar \omega$ to coincide with a zero of the Bessel function $J_{0}$.

\section{ACKNOWLEDGMENTS}

The work described in this paper was carried in collaboration with Ned Wingreen (Sections 2 and 4), Yigal Meir (Section 2), and Kristinn Johnsen (Section 3), to whom the author expresses his gratitude. A guest professorship from the Iberdrola Foundation provided delightful working conditions at ICMM in Madrid during the completition of this paper. The author is grateful to Gloria Platero and Ramón Aguado for useful comments on the manuscript. He also acknowledges the receipt of several notes, letters, corrections to incorrectly (un)quoted papers, and emails from Rolf Landauer during the last ten years or so, most of which, while perhaps critical in nature, at the same time were constructive and aimed at clarifying positions in difficult issues. Perhaps it is appropriate to end in quoting an excerpt from an email received in May 1988: ".. I am a veteran of many controversial views 
and fields; the truth in science is not reached easily, otherwise we would be unemployed. Disagreement, in print, does not mean lack of respect, and can be constructive ..... I doubt if we will come into complete intellectual equilibrium, and do not believe that is essential." 


\section{REFERENCES}

${ }^{1}$ M. Büttiker and R. Landauer, Phys. Rev. Lett. 49, 1739 (1982)

${ }^{2}$ P. K. Tien and J. P. Gordon, Phys. Rev. 129, 647 (1963)

${ }^{3}$ R. Landauer, IBM J. Res. Dev. 1, 233 (1957); Phil. Mag. 21, 863 (1970)

${ }^{4}$ M. Büttiker, Phys. Rev. Lett. 57, 1761 (1986)

${ }^{5}$ A. P. Jauho, Chapter 5 in Theory of Transport Properties of Semiconductor Nanostructures, Ed. E. Schöll, Chapman and Hall (1997) (in print)

${ }^{6}$ M. Büttiker and T. Christen, Chapter 7 in Theory of Transport Properties of Semiconductor Nanostructures, Ed. E. Schöll, Chapman and Hall (1997)

${ }^{7}$ C. A. Stafford and N. S. Wingreen, Phys. Rev. Lett. 76, 1916 (1996)

${ }^{8}$ C. A. Stafford, Phys. Rev. Lett. 77, 2770 (1996)

${ }^{9}$ T. K. Ng, Phys. Rev. Lett. 76, 487 (1996)

${ }^{10}$ A. P. Jauho, N. S. Wingreen, and Y. Meir, Phys. Rev. B 50, 5528 (1994)

${ }^{11}$ J. R. Tucker, IEEE J. of quant. electr. QE-15, 1234 (1979)

${ }^{12}$ S. Zeuner et al., Phys. Rev. B 53, 1717 (1996)

${ }^{13}$ G. Platero and Ramón Aguado, Appl. Phys. Lett. 70, 3546 (1997)

${ }^{14}$ A. Wacker et al., Phys. Rev. B, Nov. 15 (1997)

${ }^{15}$ K. Johnsen and A. P. Jauho, unpublished (archived at cond-mat/9704080)

${ }^{16}$ A. P. Jauho and K. Johnsen, Phys. Rev. Lett. 76, 4576 (1996)

${ }^{17}$ Y. Yacoby, Phys. Rev. 169, 610 (1968)

${ }^{18}$ H. Haug and S. Schmitt-Rink, Prog. Quantum Electronics 9, 3 (1984)

${ }^{19} \mathrm{~K}$. Johnsen and A. P. Jauho, unpublished. A summary of these results is found in the proceedings of OECS-5, to be published in Physica Status Solidi (1997).

${ }^{20} \mathrm{~K}$. B. Nordstrom et al. (unpublished)

${ }^{21}$ A. Yacoby et al., Phys. Rev. Lett. 74, 4047 (1995)

${ }^{22}$ R. Schuster et al., Nature 385, 417 (1997)

${ }^{23}$ A. Levy Yeyati and M. Büttiker, Phys. Rev. B 52, R14360, (1995)

${ }^{24}$ A. Yacoby, R. Schuster, and M. Heiblum, Phys. Rev. B 53, 9583 (1995).

${ }^{25}$ G. Hackenbroich and H. A. Weidenmüller, Phys. Rev. Lett. 76, 110 (1996); Phys. Rev. B, 53, 16379 (1996).

${ }^{26}$ C. Bruder, R. Fazio, and H. Schoeller, Phys. Rev. Lett. 76, 114 (1996).

${ }^{27}$ P. S. S. Guimarães et al., Phys. Rev. Lett. 70, 3792 (1993).

${ }^{28}$ L. Kouwenhoven, et al., Phys. Rev. Lett. 73, 3443 (1993).

${ }^{29}$ B. J. Keay, et al., Phys. Rev. Lett. 75, 4102 (1995).

${ }^{30}$ T. H. Oosterkamp et al., Phys. Rev. Lett. 78, 1536 (1997).

${ }^{31}$ D. Sokolovski, Phys. Rev. B 37, 4201 (1988).

${ }^{32}$ C. Bruder and H. Schöller Phys. Rev. Lett. 72, 1076 (1994).

${ }^{33}$ M. Wagner, Phys. Rev. Lett. 76, 4010 (1996).

${ }^{34}$ J. Iñarrea and G. Platero, Europhys. Lett. 34, 43 (1996).

${ }^{35}$ T. H. Stoof and Yu. V. Nazarov, Phys. Rev. B 53, 1050 (1996).

${ }^{36}$ U. Meirav and E.B. Foxman, Semicond. Sci. Technol. 10, 255 (1995).

${ }^{37}$ N. S. Wingreen, K. W. Jacobsen, and J. W. Wilkins, Phys. Rev. B, 40, 11834 (1989).

${ }^{38}$ A. P. Jauho and N. S. Wingreen, unpublished (archived at cond-mat/9706223) 